\title{
MIND, BODY AND CANCER
}

\author{
Yip CH \\ Department of Surgery, Faculty of Medicine, Univeristy of Malaya, Kuala Lumpur, Malaysia.
}

New molecular and pharmacological tools have made it possible for us to identify the intricate network that exists between the immune system and the brain, a network that allows the two systems to signal each other continuously and rapidly. Chemicals produced by immune cells signal the brain, and the brain in turn sends chemical signals to restrain the immune system. These same chemical signals also affect behavior and the response to stress. Disruption of this communication network in any way, whether inherited or through drugs, toxic substances or surgery, exacerbates the diseases that these systems guard against: infectious, inflammatory, autoimmune, and associated mood disorders. Many researchers studies have shown that stressful life events, from the death of a loved one to the loss of a job, are linked to an increase in certain health problems, particularly heart disease, diabetes, and hypertension. Many people assume that stress leads to cancer as well. Evidence for this, however, is not clear. However, the significance of mind-body interactions in medicine is now increasingly being recognised (1).

Disruption of an individual's natural sleep-wake cycle has been determined to be a contributing factor in the development of organ disease. The effect of shift work on cancer, particularly breast cancer, has received increasing interest from the lay media since a panel of the International Agency for Research on Cancer declared in 2007 that "shift work that involves circadian disruption is probably carcinogenic to humans." The majority of studies have dealt with shift work and risk of cardiovascular disease, in particular coronary heart disease, have given conflicting results possible because methodological problems are present in most studies in relation to selection bias, exposure classification, and the appropriateness of comparison groups. The study by Moy FM in this issue does not provide any evidence for a relationship between shift work and cardiovascular risk factors (2).

Cancer is the second commonest cause of death in Malaysia. A total of 21,773 cancer cases were diagnosed among Malaysians in Peninsular Malaysia in the year 2006 and registered in the National Cancer Registry.
It comprises of 9,974 males and 11,799 females. The Age standardised Incidence Rate (ASR) for all cancers in the year 2006 regardless of sex was 131.3 per 100,000 . and the five most common cancer among the population of Peninsular Malaysia in 2006 were breast, colorectal, lung, cervix and nasopharynx. A national cancer control programme on modifying risk factors for developing cancer (prevention), early detection, treatment and rehabilitation of patients with cancer is important in reducing the morbidity and mortality from this disease.

Breast cancer is the commonest cancer in women in Malaysia. Lifestyle can reduce the risk of breast cancer. Breast feeding for a prolonged period can protect against breast cancer and should be encourages. However a study on the prevalence of breast feeding in Kedah showed that only $21 \%$ of mothers breast-feed exclusively for 4 months, and women of higher education are more likely to practise breast feeding as they understand the benefits of breast feeding (3).

Occupational hazard play a small role in the causation of cancer, especially in the rare cancers such as squamous cell carcinoma of the scrotum, which has been associated with paraffin and shale oil workers (4).

Cancer may sometimes present not as the primary tumour, but as a para-neoplastic syndrome such as the oncogenic osteomalacia, characterised by bone pain and muscle weakness, and these are caused by a tumour of mesenchymal origin that secrete phosphaturic substances (5).

\footnotetext{
Correspondence:

Yip Cheng Har

Department of Surgery

Faculty of Medicine, University of Malaya

50603 Kuala Lumpur, Malaysia

Email: yipch@um.edu.my
} 
Cancer patients on chemotherapy experience psychological distress which is often under-diagnosed. This may be due to the side-effects of chemotherapy, and also the uncertainty of having a life-threatening disease. Hence it is important to recognize and treat distress early (6). Targeted therapy, which does not have the side-effects of chemotherapy, is being developed, and these drugs inhibit specific pathways involved in pathogenesis of cancer, and one such drug is temsirolimus, which is an mTOR inhibitor shown to have efficacy in renal cell carcinoma (7)

Cancer survivorship issues and quality of life are becoming increasingly important as with better and more effective treatment, women with breast cancer are living longer. Because breast cancer occurs in relatively young women, with more than $50 \%$ of women in the premenopausal age group, women with breast cancer face a multitude of problems related to sexuality. Surgery is often mutilating, chemotherapy brings on premature menopause, and there is a deterioration in sexual functioning which can post medical and psychological problems (8).

A fear of recurrence persists even after several years after a cancer is diagnosed and treated. Prognosis in breast cancer is generally good; however recurrences can occur even after many years. Clinicopathological features can be used to predict the likelihood of a relapse, and the study by Rozita AM et al in UMMC showed that disease stage, nodal status and estrogen receptor status were significantly correlated with risk of relapse (9).

Finally, we must not forget that while prescribing drugs such as chemotherapy and targeted therapy may carry side-effects, even simple drugs like antibiotics, is also not without adverse effects, and antibiotics can lead to Stevens- Johnson syndrome, and judicious use of medication with patients education is important (10).

\section{References}

1. Boey C. Reflections on the significance of relationship between mind and body in medicine. JUMMEC 2010; 13(1): 3-11.

2. Moy FM, Hoe VCW, Tan CPL, Rosmawati M. Cardiovascular risks among shift and non-shift workers in a public medical centre in Kuala Lumpur. JUMMEC 2010; 13(1): 45-49.

3. Yadav $H$. Breastfeeding practices in a rural community in Kedah. JUMMEC 2010; 13(1): 38-44.

4. Shanggar K, Ng CH, Razack HR, Dublin N. Squamous cell carcinoma of scrotum: a rare case of scrotal neoplasm. JUMMEC 2010; 13(1): 59-62.

5. Vijay P, Tan ATB, SuhaidaAM, Chan SP. Oncogenic osteomalacia, you say? Better start looking then-A Case Report. JUMMEC 2010; 13(1): 63-68.

6. Nor Zuraida Z, Ng CG. Pshychological distress among cancer patients on chemotheraphy. JUMMEC 2010; 13(1): 12-18.

7. $\mathrm{Ng} \mathrm{C.} \mathrm{A} \mathrm{retrospective} \mathrm{analysis} \mathrm{of} \mathrm{patients} \mathrm{with}$ advanced renal cell carcinoma treated withh temsirolimus. JUMMEC 2010; 13(1): 19-23.

8. Loh SY. Counselling changes in sexual functioning women with breast cancer. JUMMEC 2010; 13(1): 33-37.

9. Rozita AM, Marniza S, Mastura MY, Wan Zamaniah WI, Yip $\mathrm{CH}$. Patterns of breast cancer relapse at University of Malaya Medical Centre. JUMMEC 2010; 13(1): 24-32.

10. Rokiah Cl, Shasha K, Rokiah I. Severe cutaneous adverse drug reactions: Stevens-Johnson Syndrome and toxiv epidermal necrolysis, a report of 4 cases seen at UMMC. JUMMEC 2010; 13(1): 50-58. 